\title{
The Formation of Curved Polymer Crystals: Polychlorotrifluoroethylene
}

\author{
J. D. Barnes and F. Khoury
}

Institute for Materials Research, National Bureau of Standards, Washington, D.C. 20234

(January 29, 1974)

\begin{abstract}
The habits and fine structure of crystals of polychlorotrifluoroethylene (PCTFE) grown from dilute solution were studied as functions of crystallization temperature. The solvent used was a low molecular weight PCTFE oil. The simplest crystals found were monolayered chain folded lamellae formed at $110^{\circ} \mathrm{C}$. These lamellae are planar and possess an unusual texture characterized by the presence of fine channel-like voids in the interior of the crystals. These lamellae do not exhibit wellformed crystal faces but are disc-like in overall shape. At lower crystallization temperatures the crystals take the form of complex arrays of curved lamellae which are aggregated into, among others, watchglassshaped or hollow spherical objects. The variation of the curvature of the crystals with crystallization temperature is discussed in the light of previous studies of the formation of curved crystals of poly(4methylpentene-1) and polyoxymethylene.
\end{abstract}

Key words: Crystal morphology; crystallization; electron microscopy; optical microscopy; polychlorotrifluoroethylene; solution crystallization.

\section{Introduction}

This paper is the third in a series $[1,2]^{1}$ of three dealing with the phenomenon of curved crystal formation in polymers. The investigation we report herein stemmed from our earlier study of the crystallization of poly(4-methylpentene-1) which, as we reported in the first paper of this series [1], led to the observation that lamellar crystals of that polymer were all the more pronouncedly curved the lower the temperature (higher supercooling) at which they were grown from solution. This observation led in turn to the speculation that the manifestation of this phenomenon, rather than being peculiar to P4MP. ${ }^{2}$ might be of somewhat more general occurrence among polymers. In order to test this notion we decided to investigate other polymers. In the second paper in this series we reported that a similar phenomenon is manifested in the case of polyoxymethylene [2] crystals grown from solution.

As this paper will demonstrate folded-chain polychlorotrifluoroethylene crystals grown from solution indeed exhibit a habit-supercooling trend which, in its overall phenomenological aspects, parallels those which we have reported for P4MP and POM insofar as the manifestation of curvature and its variation in

1 Figures in brackets indicate the literature references at the end of this paper

${ }^{2}$ For the sake of brevity the names of polymers discussed in this paper will be referred to in an abbreviated form. Thus, the abbreviation for poly(4-methylpentene-1) will be P4MP, while polychlorotrifluoroethylene and polyoxymethylene will be referred to as PCTFE and POM, respectively. lamellar crystals grown at different temperatures is concerned. On the fine structural level, however, the character of PCTFE lamellae as a function of supercooling stands in marked contrast to those of P4MP and POM. This latter factor and its bearing on the origins of the dependence of the curvature of the PCTFE crystals on the conditions under which they are grown will be discussed in the light of the conjectures we have previously advanced concerning the origins of the curvature found in crystals of P4MP and POM.

By way of background to our choice of PCTFE for study it should be pointed out that we had no a priori reasons to expect curvature, or variations in curvature as a function of growth conditions, in PCTFE crystals. The choice of PCTFE was simply based on the following considerations. First, we envisaged that if curvature and variations of curvature as a function of growth conditions were manifested in crystals of a polymer, the phenomenon might be expected to manifest itself in a more complex fashion in the case of a polymer whose crystals exhibit several nonequivalent growth faces or which display a marked preference for growth along a crystallographically unique direction. Second, polymers which exist in more than one stable crystallographic phase might also be expected to exhibit more complex manifestations of this phenomenon. In line with this reasoning PCTFE which had been shown to crystallize in a hexagonal unit cell [3-7] and which does not seem to exhibit polymorphism appeared as a suitable candidate for further study, since we 
could hope to avoid the above-mentioned complications. Third, the study of PCTFE provided the attraction of uncovering some new and more detailed morphological data on the crystallization of this polymer from solution which has hitherto been poorly characterized.

\section{Experimental Details}

\subsection{Preparation of Polymer Solutions}

The powdered PCTFE polymer used in this study had a molecular weight estimated at $76,000 .^{3}$ The solvent was low molecular weight PCTFE in the form of a light oil (Kel-F Polymer Oil Grade KF-1). ${ }^{4}$ This PCTFE oil was vacuum distilled prior to use in order to remove particulate impurities.

In order to obtain solutions with a weight/volume concentration of 0.1 percent, ten milligram quantities of the powdered polymer were placed in test tubes with $10 \mathrm{~cm}^{3}$ of the PCTFE oil. The tubes were then placed in a stirred oil bath maintained at a temperature of $195{ }^{\circ} \mathrm{C}$ for one-half hour. The resulting solutions were then quickly transferred to another bath maintained at the desired crystallization temperature. Crystallization temperatures of $110,105,100$, and $90^{\circ} \mathrm{C}$ were used. Minor variations in the above regimen were necessary in the case of crystallization at $110{ }^{\circ} \mathrm{C}$. These will be described where appropriate in the text.

The onset of crystallization from the solutions (as judged by the initial appearance of cloudiness) occurred over a time span ranging from a day or two in the case of crystallization at $110{ }^{\circ} \mathrm{C}$ to a few minutes in the case of crystals precipitated from solutions placed in the $90{ }^{\circ} \mathrm{C}$ bath. After crystallization had proceeded for a long time, but before the suspensions were cooled to room temperature, the solvent containing uncrystallized polymer was isothermally replaced with fresh solvent. This replacement was accomplished as follows. First, the suspension was taken from the crystallization bath and quickly poured into a special filtration vessel akin to that described by Bassett et al. [8] situated in a bath which was maintained at the crystallization temperature. The solvent containing unprecipitated polymer was then drawn through the filter until the volume of the suspension was reduced to approximately one-fourth of its original value. Fresh solvent, preheated to the temperature of crystallization, was then added to restore the volume of the suspension to its original value. The process of reducing the volume and adding fresh solvent was then repeated at least three times. The vessel containing the crystals in suspension in fresh solvent could then be cooled to room temperature without any fear that the morphological details of the crystals would be obscured by further precipitation of polymer upon cooling the solution.

\footnotetext{
${ }^{3}$ As estimated by the manufacturer on the basis of the correlation between zero-strength time and molecular weight.

${ }^{4}$ The identification of the products of specific manufacturers is made ority for the purpose of describing experimental conditions. Such identification does not, in any way, imply endorsement by the National Bureau of Standards.
}

\subsection{Optical Microscopy}

Crystals were examined at room temperature using a phase-contrast optical microscope while they were in suspension in liquid as well as after being allowed to sediment and dry on flat glass slides. For examination of the crystals while in suspension in liquid, glass microscope slides with a shallow central depression were used. In the latter instance, liquids of appropriate refractive index (usually $n=1.58$ ) were added to the crystal suspension in order to enhance contrast.

\subsection{Electron Microscopy}

After the washed suspensions had been cooled to room temperature, droplets containing crystals were deposited on carbon films supported on copper grids and the solvent was allowed to evaporate at room temperature. Electron microscopy and electron diffraction studies were carried out on both unshadowed and $\mathrm{Pt}_{\mathrm{t}}$ : Pd shadowed crystals in a JEM 6A electron microscope. The effects of beam-induced structural changes were minimized by working at low beam intensities obtained using the double condenser system of the microscope with the second condenser lens appropriately defocused.

\section{Observations}

The morphologies of the various types of crystals formed on precipitation from 0.1 percent solutions at temperatures of $110,105,100$, and $90{ }^{\circ} \mathrm{C}$ will now be described. We shall begin with the simplest crystals found, namely those formed on crystallization at $110{ }^{\circ} \mathrm{C}$.

\subsection{Crystals Formed at $110^{\circ} \mathrm{C}$}

Crystallization in 0.1 percent solutions which were transferred directly to baths at $110^{\circ} \mathrm{C}$ was observed to proceed very slowly. Furthermore, the crystals demonstrated a marked tendency to form an almost continuous coating of intergrowing multilayered crystals adhering to the walls of the crystallization vessel. Microscopic examination of crystals formed under these conditions was very difficult. It was, however, found that "seeding" the solutions resulted in welldispersed suspensions of crystals which settled to the bottom of the crystallization vessel but could be readily stirred back into suspension and were thus more amenable to microscopic examination.

The seeding procedure which was adopted is similar in principle to that used by Blundell, Keller, and Kovacs in growing polyethylene crystals from dilute solutions at elevated temperatures [9]. Briefly, the procedure was as follows: A 0.1 percent solution of the polymer in PCTFE oil, after being held at $195^{\circ} \mathrm{C}$ for one-half hour was transferred to a bath at $80^{\circ} \mathrm{C}$. At this temperature crystallization occurs extremely rapidly, the preparation yielding complex crystals. After $45 \mathrm{~min}$ this preparation was transferred to a bath at $123{ }^{\circ} \mathrm{C}$. The cloudiness in the preparation disappeared within 5 min indicating that redissolution had occurred. The preparation was maintained at $123{ }^{\circ} \mathrm{C}$ for a total of $15 \mathrm{~min}$, at the end of which time it was transferred to 
a bath at $110^{\circ} \mathrm{C}$. Crystallization occurred within 2 days at this temperature.

The seeding procedure did not have any marked effect on the habit of the crystals grown at $110{ }^{\circ} \mathrm{C}$, other than to provide for a higher incidence of monolayer crystals of a more uniform size. Seeding of solutions crystallized at 105 and $100{ }^{\circ} \mathrm{C}$ (at which temperature it was possible to obtain crystals in suspension without resorting to seeding) had no detectable effect on the habits of the crystals formed at these temperatures.

An example of the many monolayered lamellar crystals formed at $110^{\circ} \mathrm{C}$ using the seeding procedure outlined above is shown in figure 1. The selected area electron diffraction pattern of figure 2 shows, through the presence of the six intense 10.0, and the much weaker 11.0 and 20.0 reflections (too weak to be seen in fig. 2) that the electron beam is incident along the c axis of the hexagonal unit ce'l. The unit cell parameter $a$ was calculated from the $(h k .0)$ reflections and found to be $6.42 \pm 0.03 \mathrm{~A}$, in good agreement with values of other workers [3, 4, 7]. Since the length of a typical chain (on the basis of molecular weight) is of the order of $1700 \AA$ and since the crystals are approximately only $130 \AA$ thick along the chain direction, it is clear that the chain molecules are folded in these crystals.

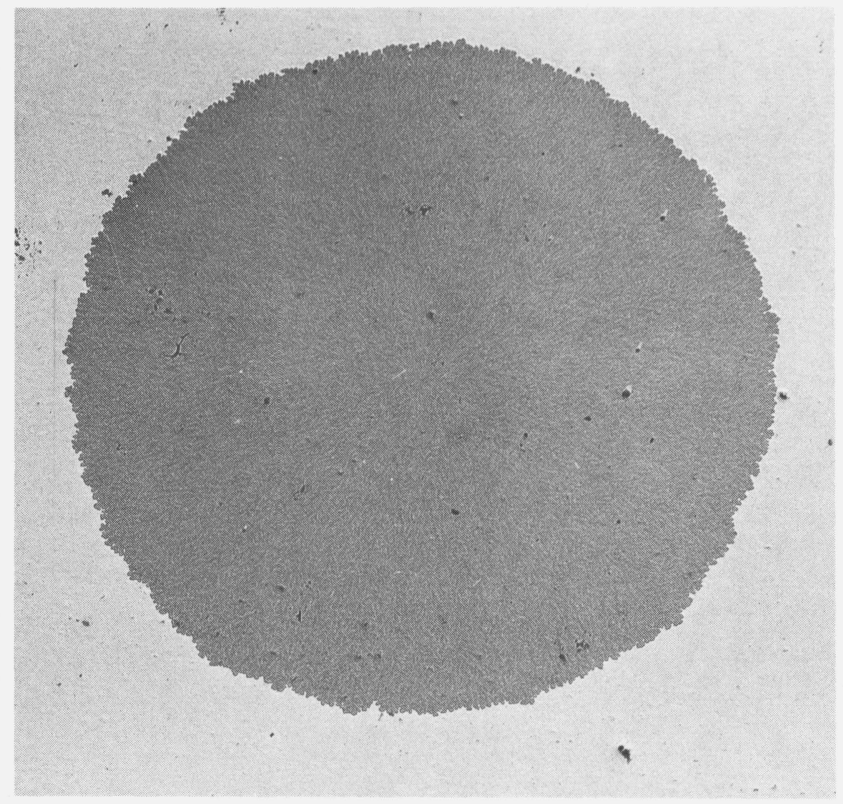

FIGURE 1. Example of disk-shaped monolayer crystal formed at $110^{\circ} \mathrm{C}$.

Note circular outline. Electron micrograph, magnification 7,900x.

Unlike chain-folded lamellar crystals of many polymers, these PCTFE crystals do not exhibit smooth, well developed, lateral growth faces. Even when formed under conditions of slow growth, as these were, chain-folded lamellae of PCTFE are essentially circular in overall shape. Furthermore, their periphery is very finely serrated.
This latter feature, which may be discerned in figures 1 and 2 and is illustrated at much higher magnification in figure 3 bears importantly on the nature of the fold domain structure in the interior of these crystals. A consideration of figure 3 clearly reveals that the fine structure of the periphery of these crystals is characterized by the profuse incidence of outgrowths (or "growth tips") (see arrow A in figure 3) of the order of $1000 \AA$ wide, which protrude outwards from the interior of the lamellae. The outgrowths often display a vague semblance of hexagonality in their outline suggesting that, on this small scale, the lateral growth pattern of the crystals may possess some semblance of crystallographic regularity.

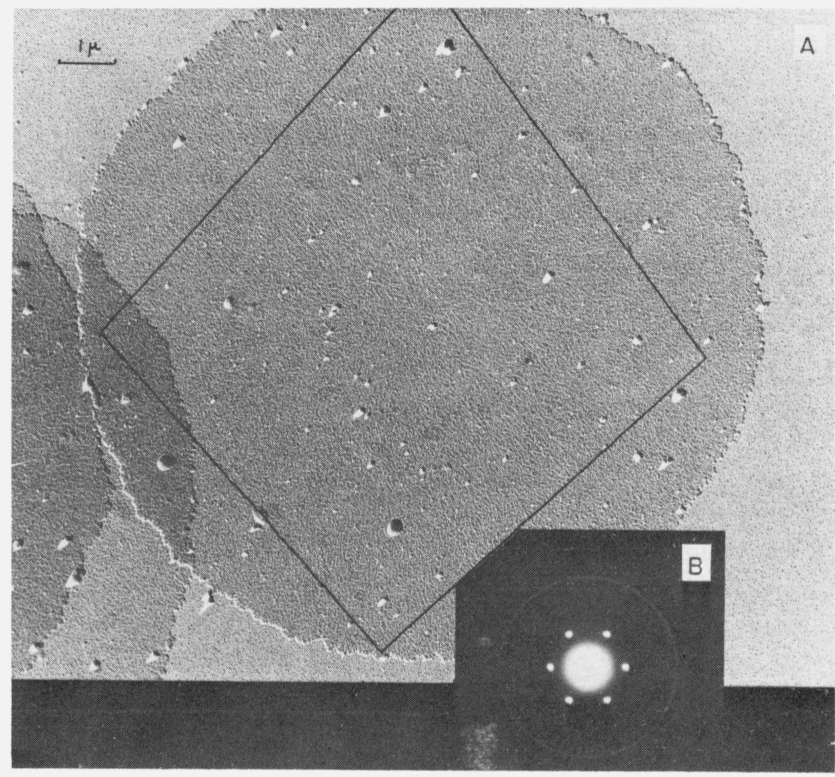

FIGURE 2. (a) Electron micrograph of monolayer crystal formed at $110^{\circ} \mathrm{C}$.

Gold shadowed. Magnification 5,700x.

(b) Selected area diffraction pattern obtained from area outlined by the black lines in (a).

The six spots are 10.0 reflections. Continuous outer ring is due to shadowing metal.

Between adjacent growth tips, as at B in figure 3, we find very narrow channels which can be traced, along meandering paths, for some distance into the interior of the crystal. In shadowed crystals the shadowing material accumulates on the sides of these channels which are away from the source, confirming that these features are indeed depressions and that they are present in the dried down crystal before it is irradiated. These channels arise because crystallizing polymer is preferentially deposited at the growth tips. Under these circumstances, the regions between the growth tips tend to become filled in more slowly. As the channels get narrower the likelihood that a molecule will diffuse into such a confined region and crystallize there becomes progressively less and the channels are never completely filled in. The unfilled channels which remain behind the growth front and become surrounded by crystalline polymer impart to 


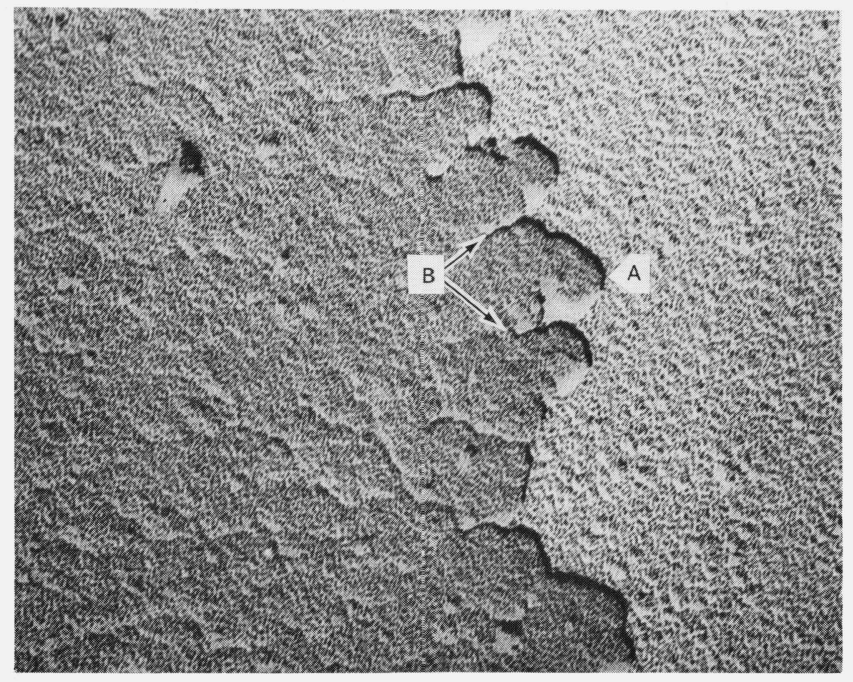

FigURE 3. High magnification view of edge of monolayered crystal formed at $110^{\circ} \mathrm{C}$.

See text for explanation of arrows. Electron micrograph, Pt:Pd shadowed. Magnification $93,000 x$.

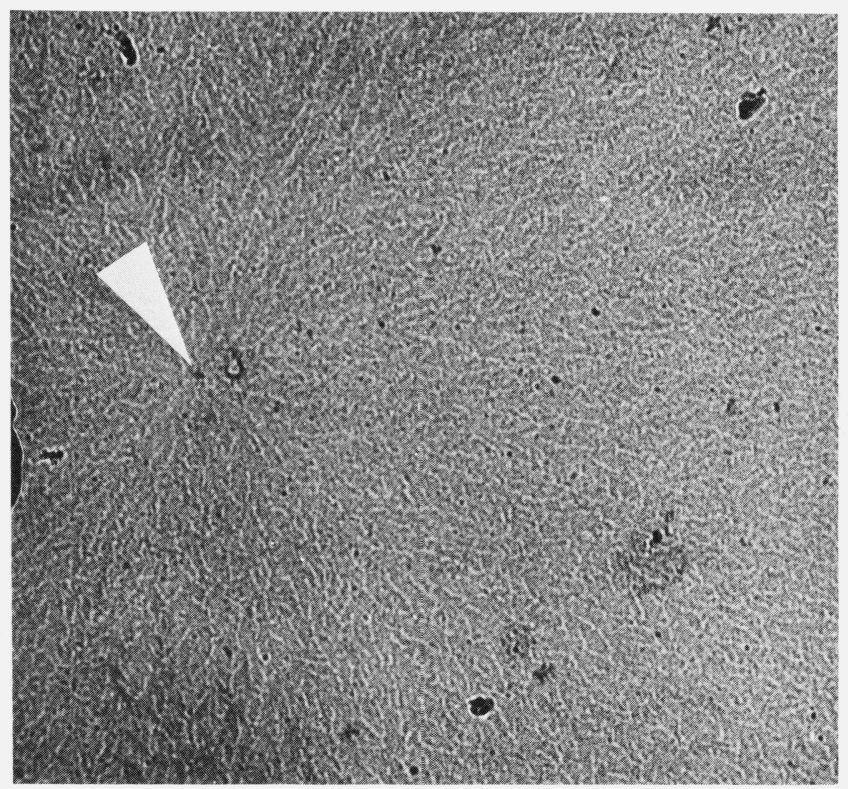

FIGURE 4. High magnification view including center of crystal grown at $110^{\circ} \mathrm{C}$

Arrow indicates center. Note radiating appearance of fine structure. Electron micrograph. Magnification 17,400x

the crystal an apparently radiating fine structure which emanates from its center. Figure 4 illustrates this feature.

This apparent radiating texture is, however, not to be interpreted as evidence for a radially symmetric internal structure. The electron diffraction pattern in figure $2 \mathrm{~b}$ indicates the monocrystal character of these lamellae. The absence of any appreciable arcing in the 10.0 reflections (as well as in the 11.0 and 20.0 reflections) cleraly indicates that the orientation of the unit cell is essentially constant throughout the crystal.

Given that the lateral growth of the crystals involves the apposition of the chain molecules in a folded conformation at the periphery of the lamellae, that their periphery is finely serrated, that they exhibit a radiating fine texture, and that no appreciable deviations in lattice orientation are observed within the lamellae, it may be readily concluded that these crystals are multisectored and consist of a crystallographically coherent finely divided mosaic of fold domains. ${ }^{5}$ The multisectored character of these crystals will be discussed in section 5.1, it may, however, be noted in anticipation of what follows that this feature and its manifestation in association with an overall essentially circular and finely serrated lateral mode of growth involving the lateral encapsulation of voids behind the growth front are characteristics of the constituent lamellae of all the crystals formed in the temperature range $110{ }^{\circ} \mathrm{C}-90{ }^{\circ} \mathrm{C}$.

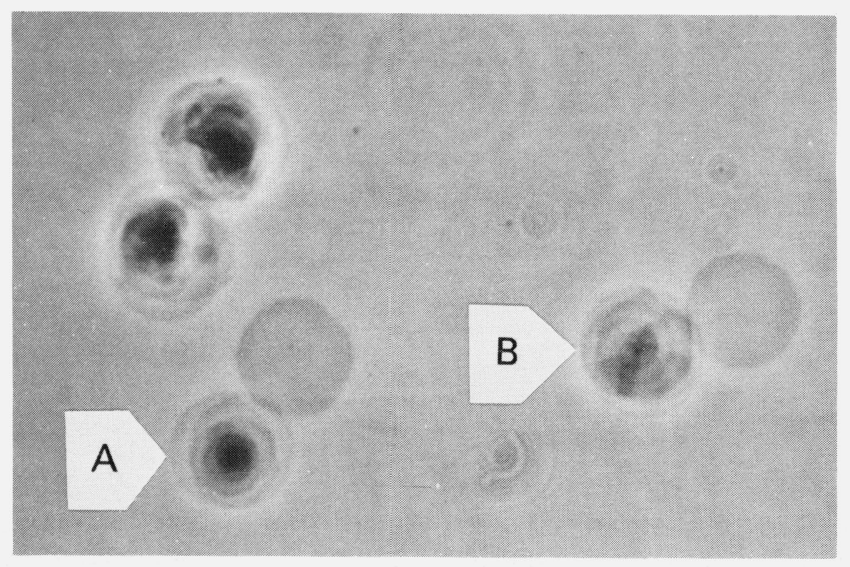

Figure $5.110{ }^{\circ} \mathrm{C}$ crystals sedimented and dried on microscope slide.

Arrows A and B point to crystals which show development of additional layers on screw dislocations. Phase-contrast optical micrograph. Magnification 1,066x.

In addition to the monolayered crystals described above, multilayered crystals whose constituent lamellae exhibit similar fine structures are also formed at $110{ }^{\circ} \mathrm{C}$. Two types of multilayered crystals were found. In the first type, examples of which are shown at (A) and (B) in figure 5, additional layers occur as spiral terraces originating from screw dislocations in the basal layer of the crystal. In the second type there are only two to four superposed layers which are of approximately equal size, and which are offset relative to one another as in the bilayer crystal shown in figure 6.

Electron diffraction patterns indicate that the superimposed layers in the bilayer crystals are rotated with respect to one another as may be seen in figure 6 . The amount of this rotation was not fixed but varied from one case to another ranging up to $15^{\circ}$ or so. It might be thought that these bilayered crystals with

\footnotetext{
The term "fold domain" and "sector" are used synonymously throughout this paper even in the absence of direct evidence linking specific crystal faces and fold domain
} boundaries. 

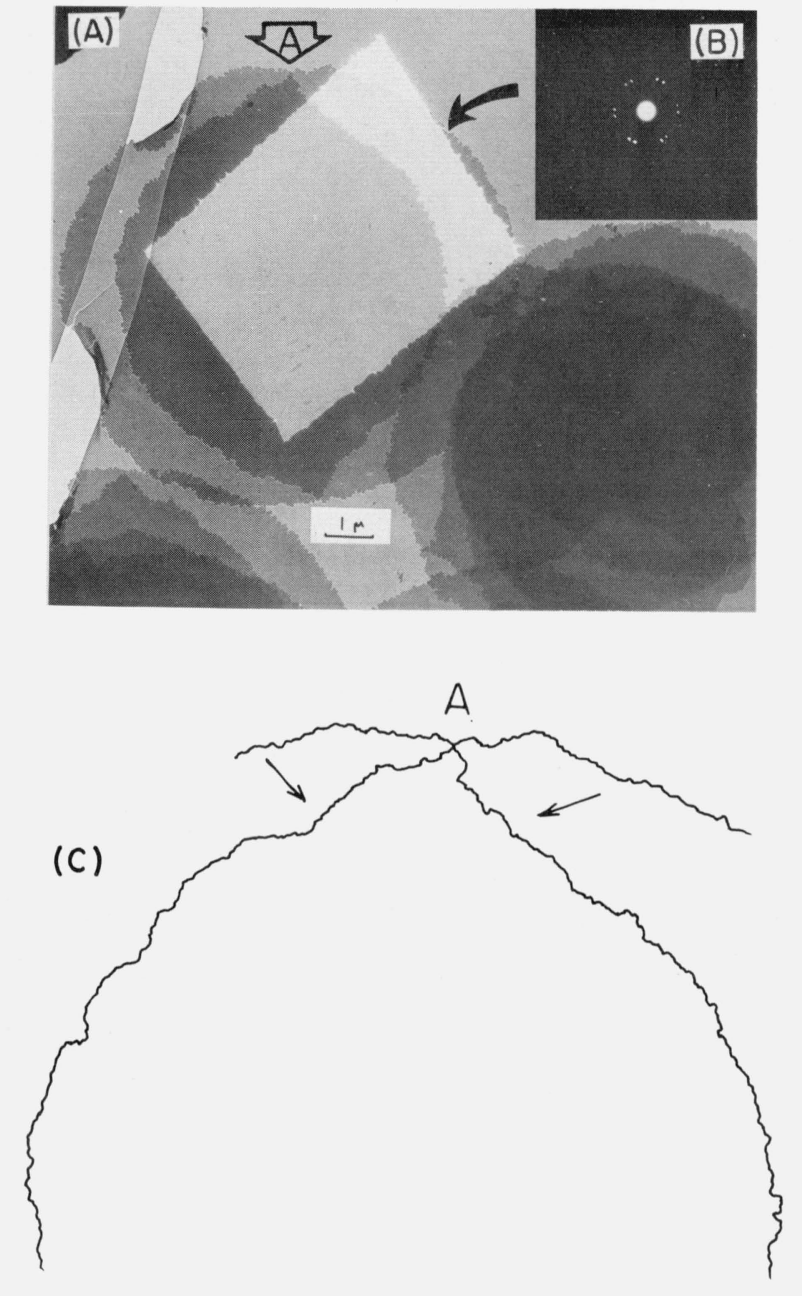

FigURE 6. Example of bilayer crystal with offset layers

(a) Example of a bilayer crystal (arrow) with offset layers. Electron micrograph, unshadowed. Magnification 5,000x.

(b) Selected area diffraction pattern obtained from lighter area outlined in (a).

Note misorientation between two sets of six 10.0 spots.

(c) Magnified schematic trace of region (A) in crystals where two layers cross.

Note that growth appears to be retarded at that portion of each lanella which is overlapped by the other layer as evidenced by the slight concavity in the vicinity of the crossover point.

apparently offset layers are merely monolayer crystals which accidently overlapped when they were deposited and allowed to dry upon a substrate. There are two features which suggest that these bilayered crystals genuinely grew as such or that they are the product of individual monolayers which impinged upon and adhered to one another (fold surface to fold surface) as they were developing in the mother liquor and subsequently proceeded to grow further in concert with one another thereafter. First, as may be seen in figure $6 \mathrm{a}$ and as shown schematically in figure $6 \mathrm{c}$ the respective peripheries of the superposed layers take on a slightly concave profile in the vicinity of the cross-over points (e.g. (A) in fig. 6a) between the overlapping layers. This feature indicates that the outward growth at the portion of the periphery of each lamella which is overlapped by the other layer tends to be slower than the outward growth at the "free" portion of the periphery of each lamella. This feature demonstrates that the two layers actually grew in concert for a substantial period of time. The second feature which confirms the specificity of this growth pattern is the frequency with which bilayered crystals occur with the two layers offset by approximately the same amount. The origins of this seemingly specific offset are at present unclear.

Having discussed the principal structural features of the $110{ }^{\circ} \mathrm{C}$ chain-folded lamellar crystals, as revealed by examination of sedimented and dried crystals, a further important aspect of their habit remains to be pointed out. This feature of these crystals, which relates to the three-dimensional character of their as-grown shapes, was investigated by examining the crystals while in suspension in liquid. They exhibit, when viewed edge-on in suspension, as are crystals $\mathrm{A}$ and $\mathrm{B}$ in figure 7 , the essentially straight-line profile to be expected of planar crystals. There is, however, a suggestion revealed in some multilayered crystals that their constituent layers may be very slightly splayed.

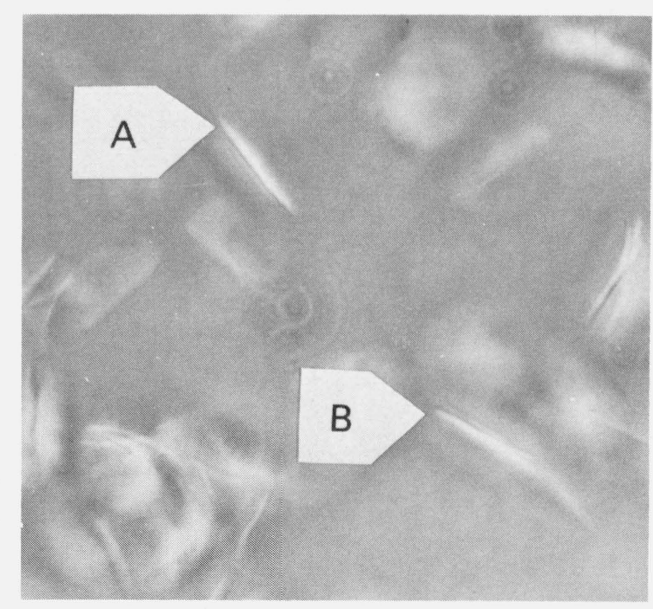

Figure 7. $110^{\circ} \mathrm{C}$ crystals in suspension in liquid.

Arrows A and B indicate crystals which are being viewed essentially edge-on. Phasecontrast optical micrograph. Magnification 1,040x.

The absence of collapse features (such as pleats, etc.) characteristic of collapsed nonplanar polymer crystals in the sedimented and dried crystals shown above, further substantiates the planar character of the chain-folded lamellae formed at $110^{\circ} \mathrm{C}$.

The planar or very nearly planar character of the crystals described above contrasts vividly with that of the crystals formed at temperatures of $105^{\circ} \mathrm{C}$ and below.

\subsection{Crystals Formed at $105{ }^{\circ} \mathrm{C}$}

A phase-contrast optical micrograph of a number of crystals formed at $105{ }^{\circ} \mathrm{C}$ as seen in suspension in liquid is shown in figure 8 in which the crescent- 
shaped edge-on profile exhibited by many of the crystals in the field of view is evident. There are actually two related types of crystals formed at this temperature.

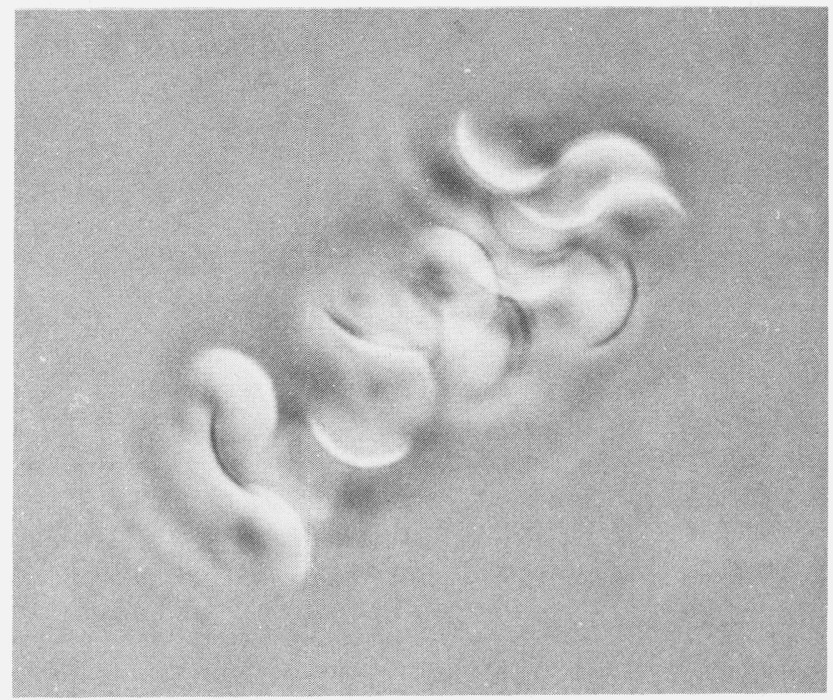

Figure 8. $105^{\circ} \mathrm{C}$ crystals in suspension in liquid.

Phase-contrast optical micrograph. Magnification 1,100x
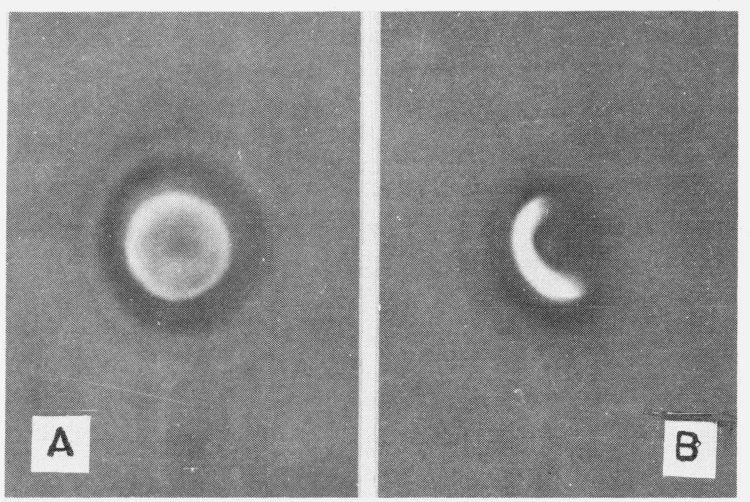

Figure 9. $105^{\circ} \mathrm{C}$ crystal in suspension in liquid.

Crystal has an overall shape approximating that of a watchglass. (a) view along central axis of crystal. (b) view perpendicular to central axis. Phase-contrast optical micrograph. Magnification 1,000x.

An example of the first, predominant, type as seen from two perspectives is shown in figure 9. This crystal exhibits, when viewed along its central axis, a circular profile as seen in figure 9a. This feature, in conjunction with the distinctly crescent-shaped profile exhibited by the same crystal when viewed edge-on, i.e., at a right angle to its central axis, as in figure 9b, demonstrates the pronouncedly curved overall shape of this species of crystal, whose shape is akin to that of a watchglass. An electron micrograph of such a crystal is shown in figure 10. These crystals consist of many superposed chain-folded lamellar layers, hence the opacity of their central regions to the electron beam; nevertheless, the lamellar character of these crystals is clearly evident in their thinner peripheral regions. The opacity of these crystals to the electron beam renders impossible the revelation, under the transmission electron microscope, of the buckling and/or folding of their constituent lamellae which occur in the central regions of the crystals as a result of their collapse upon sedimentation and drying.

The lamellar character of these crystals has already been seen in figure 10. The finely serrated character of the periphery of the constituent lamellae in these crystals can be seen more clearly at higher magnification as in figure 11. Here void-like channels extending into the interior of the crystals as well as entrapped void-like regions, both similar to those seen in the crystals formed at $110^{\circ} \mathrm{C}$, may be discerned.

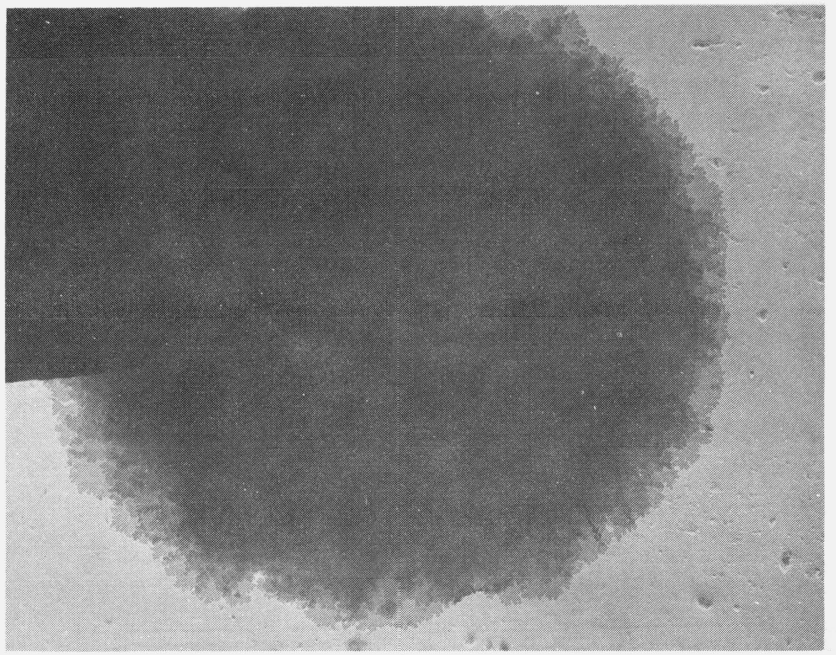

Figure $10,105^{\circ} \mathrm{C}$ crystal.

Note lamellar character. Electron micrograph magnification 5,400x.

As mentioned earlier, a second species of crystal is formed at $105{ }^{\circ} \mathrm{C}$. The as-grown shape of these crystals corresponds to that of two simple watchglass crystals such as those described above joined back-to-back such that their respective central axes are coaxial or nearly so. When viewed along their common central axis these crystals exhibit a circular periphery as is the case for the simple crystals. However, when viewed, while in suspension in liquid, along a direction normal to their common central axis, as shown in figure 12 , (arrow) they exhibit the appearance of two crescents placed back-to-back.

These multilayer crystals consist of two sets of lamellae similar in character to the constituent lamellae of the watchglass shaped crystals described earlier, the two sets of lamellae, however, being curved in opposite directions.

For the purposes of the present discussion, the salient habit feature of the crystals formed at $105^{\circ} \mathrm{C}$, which we wish to reiterate, is their distinctly curved overall shapes as contrasted with the planar character of the crystals formed at $110^{\circ} \mathrm{C}$. 


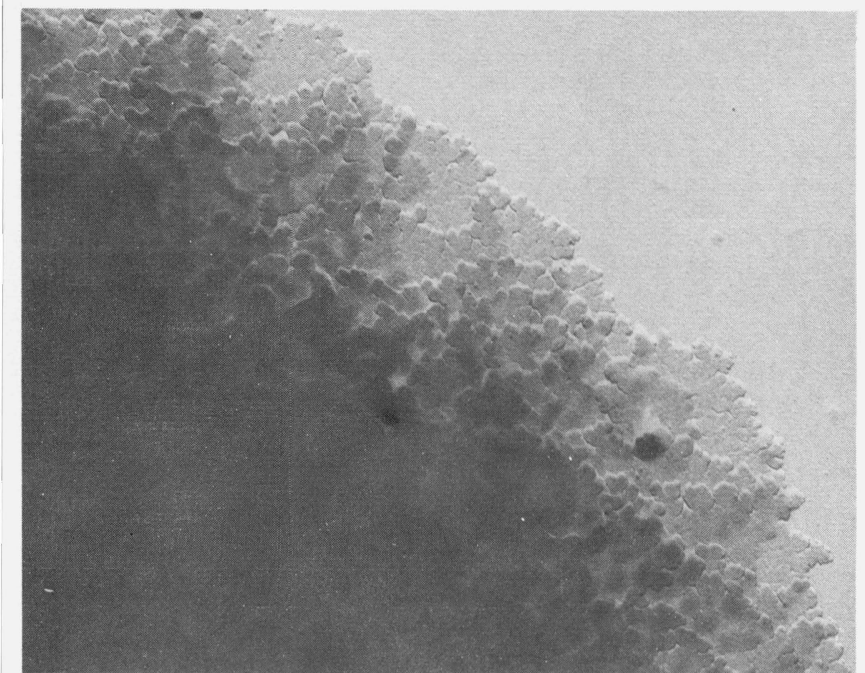

FigURE 11. High magnification view of edge of $105^{\circ} \mathrm{C}$ crystal. Note the finely serrated character of the periphery. Electron micrograph. Magnification $17,700 x$.

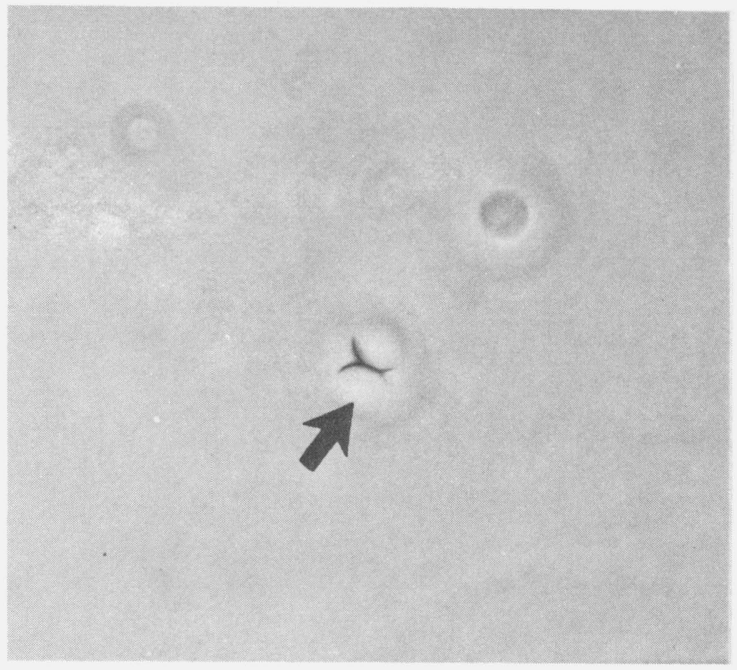

FigURE 12. Arrow points to an example of "double watchglass" type of crystal formed at $105{ }^{\circ} \mathrm{C}$.

Crystal in suspension in liquid. Phase-contrast optical micrograph. Magnification 520x.

\subsection{Crystals Formed at $100{ }^{\circ} \mathrm{C}$}

Multilayered crystals exhibiting nonplanar habits essentially similar to those of the two species grown at $105{ }^{\circ} \mathrm{C}$ are also formed when the polymer is crystallized from solution at $100{ }^{\circ} \mathrm{C}$. Two additional features may, however, be noted. First, there is a somewhat higher incidence of the double watchglass species than was observed at $105^{\circ} \mathrm{C}$, indeed, these crystals were the predominant species formed at $100{ }^{\circ} \mathrm{C}$. A phasecontrast optical micrograph depicting several such crystals (e.g., see arrows marked B) viewed edge-on or nearly so in suspension in liquid is shown in figure 13 . An example of the simpler, single watchglass crystals

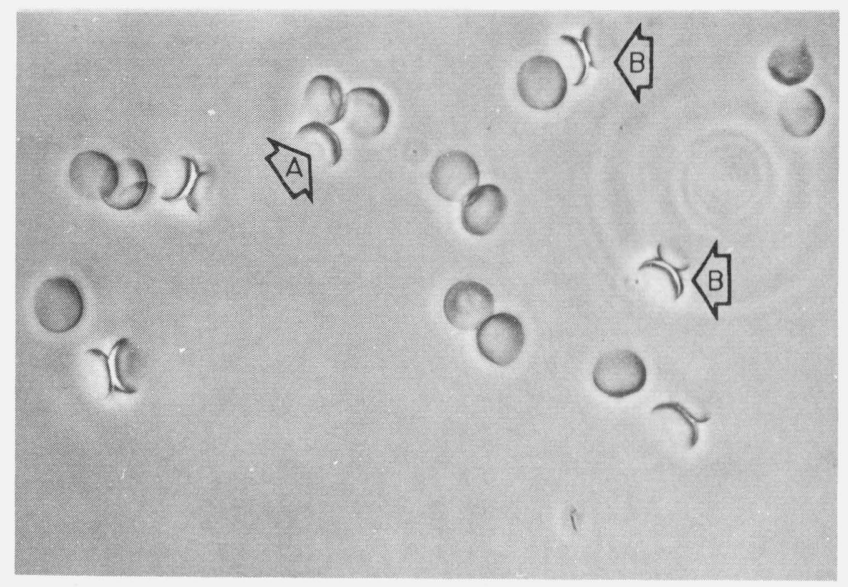

Figure 13. Group of crystals formed at $100{ }^{\circ} \mathrm{C}$ in suspension in liquid.

Arrow A points to simple watchglass-shaped crystal seen edge-on. Arrows B point to "double watchglass' crystal seen edge-on. Crystals which are edge-on in the field of view almost always have the appearance of two crescents placed back to back. Phase-contrast optical micrograph. Magnification 370x.

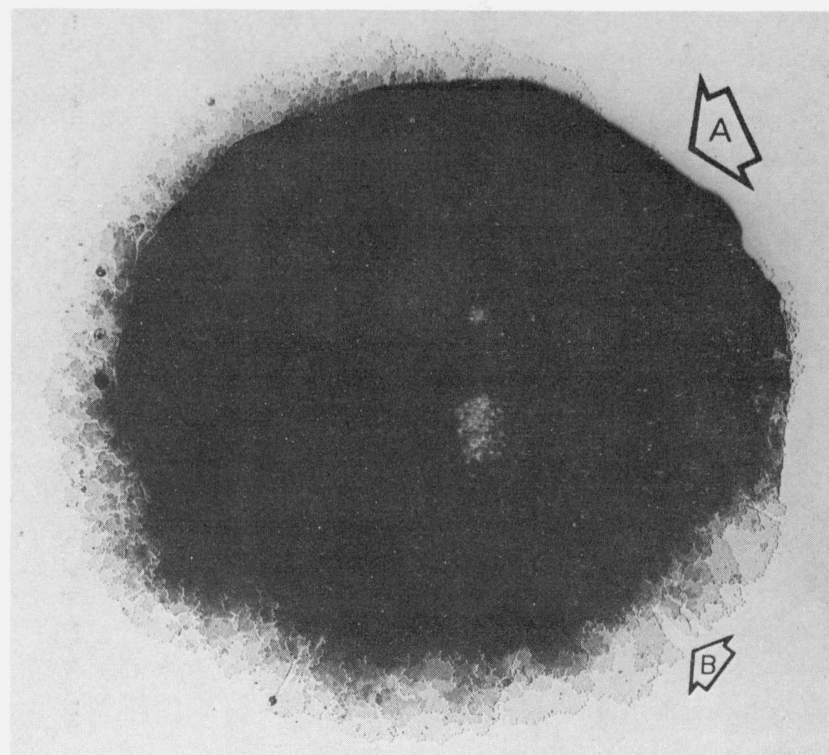

FigURE 14. $100{ }^{\circ} \mathrm{C}$ crystal.

Crystals formed at this temperature show a greater tendency to fold edges under on collapse as at A. Fracture (as at B) is also a common feature in collapsed crystals of this type. Electron micrograph. PtPd shadowed. Magnification 4,600x.

as viewed edge-on may also be seen in figure 13 (arrow marked A). Second, the shape of the single watchglass crystals and the component halves of the more complex, double watchglass, species appear to be more hemispherical than watchglass-like. This feature, though to some extent subjective, is partially substantiated by the greater frequency with which the lamellae at the crystal periphery tend to fold inwards upon sedimentation and collapse as shown in region $\mathrm{A}$ in the electron micrograph of a collapsed crystal illustrated in figure 14. Fracture (see B in fig. 14) is a feature which often occurs when crystals of this type 


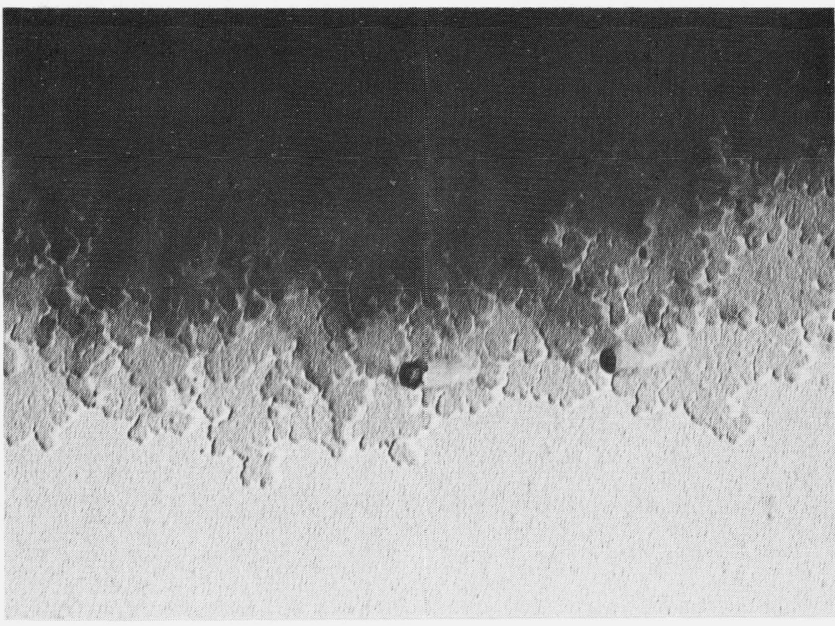

Figure 15. High magnification view of periphery of $100^{\circ} \mathrm{C}$ crystal showing highly reentrant character of the lamellae.

Electron micrograph. magnification $19,700 x$.

collapse. The resulting cracks, when viewed at high magnification, are seen to be traversed by fine fibrils. Figure 14 reveals the lamellar character of these crystals and the finely serrated character of the periphery of their constituent lamellae. These two features are more clearly revealed in figure 15 which is a highly magnified view of the outer portion of a crystal. Figure 15 also reveals the similarity in the nature of the constituent lamellae in the crystals formed at $100{ }^{\circ} \mathrm{C}$ and those formed at 105 and $110^{\circ} \mathrm{C}$.

\subsection{Crystals Grown at $90{ }^{\circ} \mathrm{C}$}

Because growth is very rapid at temperatures of $90{ }^{\circ} \mathrm{C}$ and below some crystallization takes place before temperature equilibrium is attained when solutions initially held at $195{ }^{\circ} \mathrm{C}$ are transferred to baths at $90{ }^{\circ} \mathrm{C}$. The phase contrast micrograph shown in figure 16 illustrates the appearance of crystals formed when a solution is cooled to $90{ }^{\circ} \mathrm{C}$. In the multilayered crystals, denoted A in figure 16, the lamellae have curved around to completely enclose the hollow centers of the crystals in an onionskin fashion. Birefringence observations show that the chain molecules are parallel to the radius vector in these hollow, essentially spherical, crystals.

More complex crystals, such as those denoted B in figure 16 are also formed under these conditions. These crystals are complex arrays of curved chain-folded lamellae. The origin of these arrays may readily be envisaged as the consequence of a pronounced tendency for fresh layers, initiated on a curved parent lamella. The origin of these arrays may readily be parent lamella at their point of origin. This effect is similar to that already observed on a more limited scale in the double watchglass crystals seen at 105 and $100{ }^{\circ} \mathrm{C}$.

Since they consist of many chain-folded layers, the crystals formed at $90{ }^{\circ} \mathrm{C}$ are too thick to examine in direct transmission in the electron microscope. We

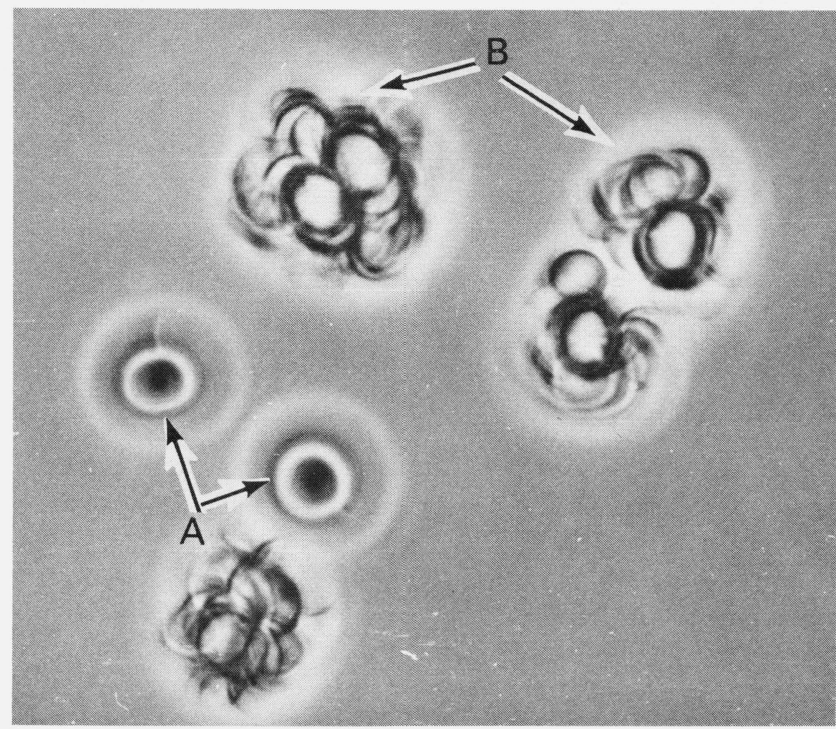

Figure 16. Crystals formed at $90{ }^{\circ} \mathrm{C}$ in suspension in liquid.

The arrows marked A indicate hollow spherical crystals in which curved lamellae completely enclose the centers. The arrows marked B point to examples of the many complex arrays of curved lamellae found at this temperature. Phase-contrast optical micrograph. Magnification $840 \mathrm{x}$.

therefore resorted to replication techniques in order to examine the fine structure of these crystals. Figure 17 shows a replica of a crystal from a suspension cooled to $90{ }^{\circ} \mathrm{C}$. These replicas were prepared by evaporating a Pt:Pd alloy onto crystals which had been sedimented onto a flat glass microscope slide and dried there. Carbon was then evaporated over the $\mathrm{Pt}: \mathrm{Pd}$ layer to provide a backing. The film of $\mathrm{Pt}: \mathrm{Pd}$ and carbon containing the crystals was then cut into small squares and floated off the glass slide onto a water surface. The small squares were then picked up and dried on electron microscope grids. After drying, the grids were immersed in a vessel containing PCTFE oil at room temperature. This vessel was then slowly heated up to $175{ }^{\circ} \mathrm{C}$ in order to dissolve the polymer and extract it from the replica.

The electron micrograph shown in figure 17 reveals the collapse features formed on sedimentation of these nonplanar structures. In order to see the fine structural characteristics of the lamellae the replica must be examined at higher magnification, as in figure 18. In figure 18 the serrated character of the periphery of the lamellae is clearly visible, thus showing the similarities between the lamellae of which these crystals are composed and those formed at the higher temperatures.

\section{Summary of Results}

The observations described above provide a detailed characterization of the crystals which are observed to form when PCTFE is precipitated from dilute solutions at a number of temperatures in the range from 110 to $90{ }^{\circ} \mathrm{C}$. The habits of the crystals are observed to depend strongly on the degree of supercooling prevailing during their growth.

At $110^{\circ} \mathrm{C}$ we find disk-like monolayered, chain folded lamellae exhibiting an essentially planar overall shape. 


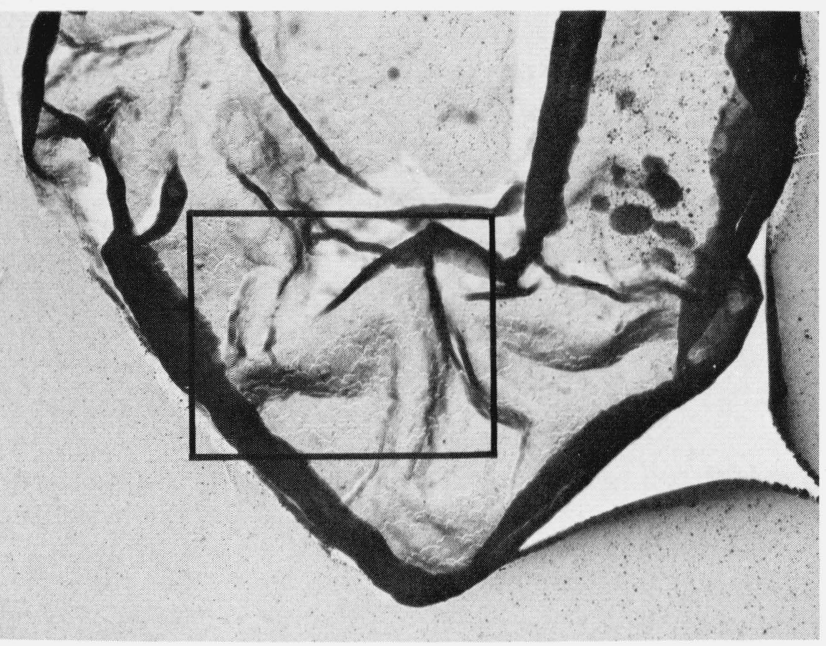

FIGURE 17. Shadow-transfer replica of a portion of a crystal formed at $90{ }^{\circ} \mathrm{C}$, permitting the observation of the buckling and pleating resulting from collapse of the crystals.

Electron micrograph. Magnification 10.300x.

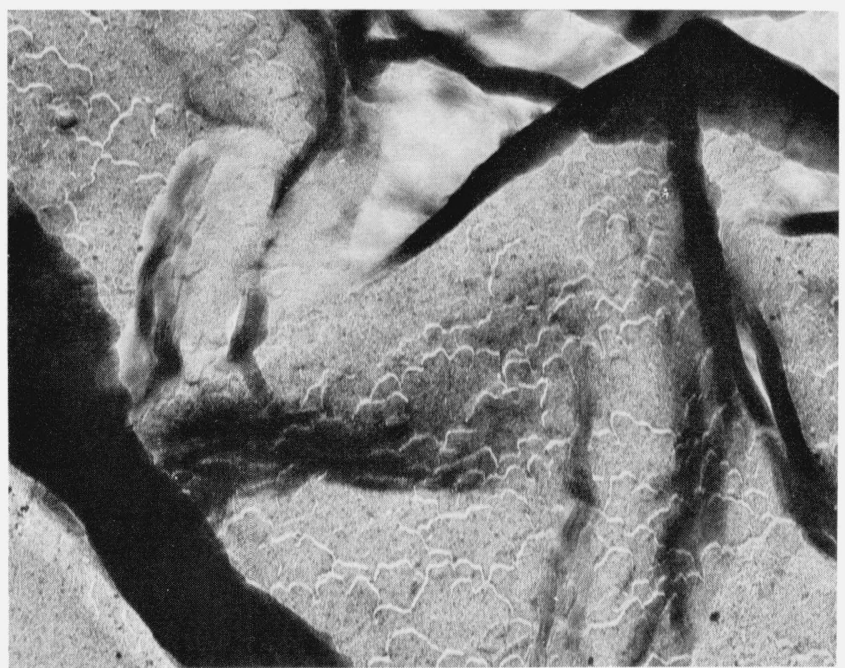

FIGURE 18. Higher magnification view of region outlined in figure 17.

Note the lamellar texture of the surface and the highly serrated character of the lamellae. Electron micrograph. Magnification $28,000 x$.

These unusual crystals are characterized by an absence of prismatic growth faces and the presence of a highly multisectored fine structure. Although this growth pattern suggests that the lamellae possess an irregular internal structure, electron diffraction reveals that these lamellae have a single-crystal character with the orientation of the unit cell remaining essentially constant throughout the crystal. Multilayered crystals are also formed at $110{ }^{\circ} \mathrm{C}$. The constituent lamellae in these crystals are similar to the monolayered crystals.

The crystals which are formed at somewhat higher degrees of supercooling, i.e., upon crystallization at $105^{\circ} \mathrm{C}$ and at $100^{\circ} \mathrm{C}$ are of two types. The simpler ones possess a curved overall shape akin to that of a watchglass. The shape of the more complex corresponds to that of two watchglasses joined back to back. These structures are multilayered, consisting of stacks of curved lamellae. At even higher degrees of supercooling we find, along with other, more complex, structures, crystals in the form of hollow spheres where the curving lamellae completely enclose the hollow centers of the crystals in an onion-skin fashion.

Crystals formed at $105^{\circ} \mathrm{C}$ have a radius of curvature in the range $5-6 \mu \mathrm{m}$. Those formed at $100{ }^{\circ} \mathrm{C}$ have a slightly smaller radius of curvature and those formed at $90{ }^{\circ} \mathrm{C}$ have a radius of curvature in the range 4-4.5 $\mu \mathrm{m}$. This variation is much less than that found in our earlier studies [1,2].

Paralleling the trend toward increasingly curved crystals as the degree of supercooling is increased we find an increasing tendency for new layers, initiated on a growing crystal, to adopt a sense of curvature opposite to that of the parent lamellae. This tendency leads to the formation, at high degrees of supercooling, of complex pseudospherulitic arrays of curved lamellae (see fig. 16).

\section{Discussion}

For the purposes of discussion we will group the results of our experiments into two classes. In the first instance we will discuss the fine structural features observed in the simple monolayered crystals formed at $110{ }^{\circ} \mathrm{C}$. These features are representative of those exhibited by the lamellae of which crystals formed at other temperatures are composed. As a second area for discussion we will consider the observed effects of crystallization temperature on the overall shapes of PCTFE crystals.

\subsection{Fine Structure of Lamellar Crystals of PCTFE}

The important features of the fine structure of the PCTFE folded chain lamellae we have observed in this study are best exemplified in the monolayered chain-folded crystals formed at $110{ }^{\circ} \mathrm{C}$. Turning our attention to the overall appearance of these crystals we see that, instead of being bounded by smooth crystallographic growth faces, they exhibit an essentially circular outline. This feature, together with the apparently radiating structure caused by channel-like voids in the interior of the crystals and the finely serrated character of the periphery, suggests that the crystals grow in an irregular manner. In characterizing and attempting to account for this irregular growth pattern, we must however, bear in mind that it leads to a structure which is crystallographically coherent; i.e., the unit cell orientation is essentially constant throughout the crystal.

If we examine the influence of crystallization temperature on crystal habit we see differences in behavior pattern between PCTFE and such "normal" polymers as P4MP and POM which require some comment. In these latter cases crystals with welldefined growth faces and consisting of only a few fold domains are formed under conditions where growth is slow. As crystals are formed at lower and lower temperatures, favoring increasingly rapid growth, the lamellae develop in a multisectored manner, 
i.e., they are subdivided into an increasingly finer mosaic of fold domains due to microfaceting associated with the development of successive arrays of sectored outgrowths. In the case of PCTFE, however, the profile of the growing face remains essentially unchanged as growth becomes more rapid and the influence of growth rate on sector size is not nearly so marked as in P4MP and POM.

In order to discover whether the solvent was a factor influencing the fine structure of PCTFE crystals we attempted to crystallize the polymer from mesitylene and from 2,5-dichlorobenzotrifluoride. These experiments yielded morphologies essentially the same as those obtained on crystallization from PCTFE oil. Our experimentation in an attempt to obtain crystals of PCTFE with well-defined prismatic faces has been far from exhaustive and, since it is known that parameters such as solvent, molecular weight, concentration, and temperature affect morphology, it will be necessary to carry out further experiments in order to establish whether or not PCTFE can be induced to form regular prismatic crystals under suitably chosen conditions.

A factor in addition to those mentioned above which might influence the habits of PCTFE crystals can be found in the steric character of the molecules. This effect would be an intrinsic one arising from the molecular conformation and could be relatively unaffected by the coice of crystallization conditions. In the case of PCTFE the $\mathrm{CF}_{2} \mathrm{CFCl}$-groups follow each other in a random sequence with syndiotactic diads occurring approximately twice as frequently as isotactic ones [10]. This irregular placement of chlorine atoms may lead to an irregular lateral packing of the molecules. ${ }^{5} \mathrm{We}$ do not, as yet, have a model linking such an effect to the observed crystal habit and further experimentation is necessary to establish the importance of steric factors in regulating interchain packing and thereby possibly influencing crystal habits.

Having indicated some possible origins of the irregular lateral habit of crystals of PCTFE let us return for a moment to some questions relating to the process of chain folding in this polymer. From the fact that the length of any given molecule is, on the average, much greater than its extension in the crystal we must conclude that the molecular chains in these crystals are folded. Although one would suspect that the molecules could fold in a regular manner along (10.0) or possibly even (11.0) planes we have been unable, because of the lack of clearly distinguishable prismatic facets in these crystals, to identify any specific sets of planes as containing the molecular chain folds.

Lacking an answer to this question it is impossible to characterize the fine structure of these crystals in terms of specific fold domains (or sectors). This question could, or course, be resolved by characterizing well-formed crystals and observing the process of degeneration into the habit described above. Since we have not yet attained this goal we will content ourselves, in the following discussion, with using terms like "fold domain" and "domain structure" as a short-

\footnotetext{
${ }^{5}$ This question is investigated more fully in the recent study by Mencik [7].
}

hand description for the fine structure of the crystals even though the relationship between this fine structure and the chain folding process is not clear.

\subsection{Curved Crystal Formation in PCTFE}

In our earlier papers [1,2] we advanced, as a working hypothesis, a model which is presumed to account for curved crystal formation in P4MP and POM through the influence of two factors. First, detailed diffraction contrast observations on crystals of these two polymers indicate that individual fold domains are nonplanar. Thus, a simple four or six-sectored crystal is actually slightly conical in overall shape. This degree of conicalness is extremely small and is most readily assessed by means of diffraction contrast experiments on simple crystals composed of only a few fold domains. The second factor influencing curvature is the nature of the interconnection between adjoining fold domains in a multisectored crystal. We suppose that adjacent fold domains are joined together in a coherent manner such that their nonplanarity accumulates. On this basis we expect a multisectored crystal of a given size to be more highly curved than one possessing only a few fold domains. In actual fact the phenomenology of curved crystal formation is more complicated than this and we would strongly advise the interested reader to consult the more detailed discussion in reference [1].

From the phenomenological point of view this description fits the observed characteristics of the crystals rather well. In the case where crystals of P4MP and POM consisted of only a few fold domains we found essentially planar crystals whereas highly multisectored crystals were found to be curved. The crystals of PCTFE formed at temperatures below $110^{\circ} \mathrm{C}$ also fit this pattern in that they are both strongly curved and highly multisectored.

PCTFE crystals formed at $110^{\circ} \mathrm{C}$ do not fit into the previously established pattern for curved crystal formation because they are essentially planar in their overall shape in spite of the fact that they are highly multisectored. On the basis of this observation we might, within the hypothetical framework described above, surmise that fold domains in $110^{\circ} \mathrm{C}$ crystals are planar or that there is insufficient connectivity between adjoining fold domains to allow neighboring non-planar fold domains to make a cumulative contribution to the overall curvature of the crystal.

On the basis of our experiments we cannot determine which, if either, of these effects is present. In the first place we have been unable to obtain diffraction contrast effects which allow an assessment of the nonplanarity of individual fold domains. Such an undertaking would be difficult, if not impossible, on crystals such as ours because of the very small size of the fold domain structure. It is even less likely that one could measure, by means of Moire patterns [11], lattice distortions which could give rise to nonplanar fold domains in such crystals.

Since such information is lacking we cannot assert with any positiveness that curved crystal formation is 
a phenomenon which is caused by the same factors in the three polymers we have examined. The fact that crystals formed at lower temperatures are both curved and multisectored in all three cases does permit us to speculate that this is so. In this context and in order to fit the observations on PCTFE crystals formed at $110{ }^{\circ} \mathrm{C}$ into the phenomenological framework of our earlier work we would then further speculate that:

(1) Fold domains in PCTFE crystals formed at low supercoolings are more nearly planar than those in crystals formed at high supercoolings or, (2) that in PCTFE crystals formed at low supercoolings there is insufficient connectivity between neighboring nonplanar fold domains to allow for the propagation of a curved structure, while at high supercoolings the nonplanarity of neighboring fold domains contributes to overall curvature in a cumulative manner.

We can summarize our discussion of curved crystal formation in PCTFE by noting that, while PCTFE does indeed exhibit a habit-supercooling trend parallelling that exhibited by P4MP and POM, we are unable to characterize the possible origins of this trend in PCTFE. If suitable crystals were available it might be possible to make more affirmative statements about the origins of curvature in PCTFE crystals.

Finally, the observation that increasing supercooling tends to favor the development of arrays of lamellae which curve in opposite directions as in the double watchglass shaped crystals formed at $100{ }^{\circ} \mathrm{C}$ and the complex structures (e.g., B in fig. 16) formed at $90{ }^{\circ} \mathrm{C}$, bears further comment. The conclusion we wish to point out in this connection is the following: The curvature of the constitutent lamellae in those crystals is an intrinsic feature of the lamellae and is not caused by repulsive interactions between superposed lamellae as might be invoked to account for the splaying of the lamellae in opposite directions. We base this conclusion on the fact that curvature is an intrinsic feature of the single watchglass shaped crystals formed at 105 and $100{ }^{\circ} \mathrm{C}$ as well as the hollow spherical shaped crystals formed at $90{ }^{\circ} \mathrm{C}$ which are curved in only one direction.

\section{Conclusions}

Crystals of PCTFE were obtained by crystallizing the polymer from 0.1 percent solutions of PCTFE at temperatures of $110,105,100$, and $90{ }^{\circ} \mathrm{C}$. At $110{ }^{\circ} \mathrm{C}$ both monolayered and multilayered chain-folded lamel- lar crystals were formed. The crystals were planar. The individual lamellae exhibited an essentially circular overall outline and their periphery was finely serrated. The internal fine structure of the lamellae is mosaiclike on a very fine scale. The irregular character of the lateral mode of propagation of the lamellae and their complex internal texture prevents any firm statements regarding the chain-folding process involved in the formation of these crystals.

At lower crystallization temperatures multilayered crystals exhibiting curved overall shapes were formed. The curvature of these crystals tended to be more pronounced the lower the crystallization temperature. This effect is not nearly as strong as those observed for P4MP and POM [2]. The fine structures of the lamellae in these curved crystals was however similar to that of those formed at $110{ }^{\circ} \mathrm{C}$. The origins of the fact that the crystals of PCTFE tended to be all the more pronouncedly curved the lower the crystallization temperature remain obscure at this stage. Uncertainties regarding the nature of the fold domain structure in the crystals preclude us from making any connection between our present study and our previous ones on the formation of curved crystals of P4MP and POM other than the phenomenological one that (as was the case for P4MP and POM) the PCTFE crystals tended to be all the more pronouncedly curved the lower the crystallization temperature.

\section{References}

[1] Khoury, F., and Barnes, J. D., J. Res. Nat. Bur. Stands. (U.S.), 76A (Phys. and Chem.), No. 3, 225-252 (May-June 1972).

[2] Khoury, F., and Varnes, J. D., J. Res. Nat. Bur. Stands. (U.S.), 78A (Phys. and Chem.), No. 2, 95-127 (Mar.-Apr. 1974).

[3] Kaufman, J. S., J. Am. Chem. Soc. 75, 1477 (1953).

[4] Ermolina, A. V., Markova, G. S., and Kargin, V. A., Soviet Physics - Crystallography 2, 615 (1957).

[5] Roldan, L. G., and Kaufman, H. S., Norelco Reporter 10, \#1, 11 (1963).

[6] Miyamoto, Y., Nakafuku, C., and Takemura, T., Polymer Journal (Japan) 3, 122 (1972).

[7] Mencik, Z., J. Polymer Sci. Pt. A-2, 11, 1585 (1973).

[8] Bassett, D. C., and Keller, A., Phil. Mag. 7, 1553 (1962).

[9] Blundell, D. J., Keller, A., and Kovacs, A. J., J. Polymer Sci. B4, 481 (1966).

[10] Bovey, F. A., and Tiers, G. V. D., Fortschr. Hochpolym. Forsch. 3, 139 (1963).

[11] Bassett, D. C., Phil. Mag. Ser. VIII 10, 595 (1964).

(Paper 78A3-817) 